Weinert, Franz E.

\title{
Begabung und Lernen. Zur Entwicklung geistiger Leistungsunterschiede
}

Hackl, Armin [Hrsg.]; Pauly, Claudia [Hrsg.]; Steenbuck, Olaf [Hrsg.]; Weigand, Gabriele [Hrsg.]: Werte schulischer Begabtenförderung. Begabung und Leistung. Frankfurt, M. : Karg-Stiftung 2012, S. 23-34. (Karg-Hefte. Beiträge zur Begabtenförderung und Begabungsforschung; 4)

Quellenangabe/ Reference:

Weinert, Franz E.: Begabung und Lernen. Zur Entwicklung geistiger Leistungsunterschiede - In: Hackl, Armin [Hrsg.]; Pauly, Claudia [Hrsg.]; Steenbuck, Olaf [Hrsg.]; Weigand, Gabriele [Hrsg.]: Werte schulischer Begabtenförderung. Begabung und Leistung. Frankfurt, M. : Karg-Stiftung 2012, S. 23-34 URN: urn:nbn:de:0111-opus-90291 - DOI: 10.25656/01:9029

https://nbn-resolving.org/urn:nbn:de:0111-opus-90291

https://doi.org/10.25656/01:9029

in Kooperation mit / in cooperation with:

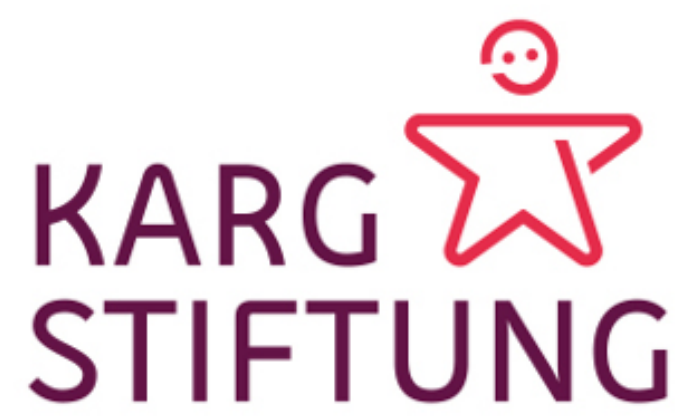

https://www.karg-stiftung.de

https://www.fachportal-hochbegabung.de

\section{Nutzungsbedingungen}

Gewährt wird ein nicht exklusives, nicht übertragbares, persönliches und beschränktes Recht auf Nutzung dieses Dokuments. Dieses Dokument ist ausschließlich für den persönlichen, nicht-kommerziellen Gebrauch bestimmt. Die Nutzung stellt keine Übertragung des Eigentumsrechts an diesem Dokument dar und gilt vorbehaltlich der folgenden Einschränkungen: Auf sämtlichen Kopien dieses Dokuments müssen alle Urheberrechtshinweise und sonstigen Hinweise auf gesetzlichen Schutz beibehalten werden. Sie dürfen dieses Dokument nicht in irgendeiner Weise abändern, noch dürfen Sie dieses Dokument für öffentliche oder kommerzielle Zwecke vervielfältigen, öffentlich ausstellen, aufführen, vertreiben oder anderweitig nutzen.

Mit der Verwendung dieses Dokuments erkennen Sie die Nutzungsbedingungen an.

\section{Terms of use}

We grant a non-exclusive, non-transferable, individual and limited right to using this document.

This document is solely intended for your personal, non-commercial use. Use of this document does not include any transfer of property rights and it is conditional to the following limitations: All of the copies of this documents mus retain all copyright information and other information regarding legal protection. You are not allowed to alter this document in any way, to copy it for public or commercial purposes, to exhibit the document in public, to perform, distribute or otherwise use the document in public.

By using this particular document, you accept the above-stated conditions of use.

\section{Kontakt / Contact:}

peDOCS

DIPF | Leibniz-Institut für Bildungsforschung und Bildungsinformation Informationszentrum (IZ) Bildung

E-Mail: pedocs@dipf.de

Internet: www.pedocs.de

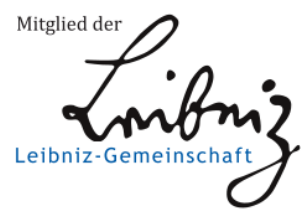


Editorial:

"Leistung muss sich wieder lohnen « - oder eine Geburtsstunde der Hochbegabtenförderung

Ingmar Ahl

Begabung und Leistung: Zur Einführung

Gabriele Weigand, Olaf Steenbuck, Claudia Pauly,

Armin Hackl

Begabungsbegriff und Leistung.

Eine pädagogische Annäherung

Timo Hoyer

Begabung und Lernen.

Zur Entwicklung geistiger Leistungsunterschiede

Franz E. Weinert

Zum Verhältnis von Begabung und Leistung

Ernst A. Hany

Herausragende Leistungen durch Lust \& Leidenschaft im Kreativen Feld

Olaf-Axel Burow

Leistung heißt Kompetenzentwicklung

Klaus Amann

Leistung gehört zur Schule wie das

Mittagessen zum Kochen!

Oder: Wie viel Mittagessen verträgt der Mensch?

Ursula Hellert

Leistung in Konzepten der Hochbegabtenförderung

Richard Greiner, Ulrich Halbritter

Reflexivität und Leistung

Claudia Pauly, Gabriele Weigand

Sozialität und schulische Leistung. Überlegungen zu einem erweiterten Leistungsbegriff

Insa Martin, Annette von Manteuffel

Autonomie und Leistung

Werner Esser

Leistung und Hochbegabung. Überlegungen zu einem personalen Leistungsverständnis

Armin Hackl 


\section{Begabung und Lernen}

\section{Zur Entwicklung geistiger Leistungsunterschiede ${ }^{1}$}

Manche werden die Überschrift dieses Vortrags für anmaßend halten, ist sie doch mit dem Titel eines Buches identisch, das vom Deutschen Bildungsrat 1969, also vor genau dreißig Jahren, veröffentlicht wurde. Es hat das deutsche Schulsystem bis heute nachhaltig beeinflusst. Einige der in diesem Band enthaltenen Gutachten dienten nämlich als wissenschaftliche Begründung oder Rechtfertigung für revolutionäre Veränderungen des Bildungswesens in vielen Ländern der Bundesrepublik Deutschland.

Der damalige Vorsitzende der Bildungskommission im Deutschen Bildungsrat Karl Dietrich Erdmann kennzeichnete schon in seinem Vorwort die neue bildungstheoretische Vision: „Wenn für die Erfüllung jeweiliger Lernanforderungen adäquate Begabung Voraussetzung ist, so gilt nach den Aussagen dieses Gutachtenbandes noch mehr der umgekehrte Satz, dass im Zusammenwirken der Faktoren, durch die Begabung zustande kommt und sich entwickelt, die richtig angelegten Lehr- und Lernprozesse selbst entscheidende Bedeutung besitzen ( Erdmann, 1969, 5f.).

»Man ist nicht begabt, sondern man wird begabt«, wurde zur hoffnungsvollen Maxime, später zur verzweifelten Hoffnung ungezählter Lehrer; die Egalisierung der geistigen Entwicklung unterschiedlicher Menschen durch kompensatorische Bildung wurde zum fundamentalen Ziel vieler Politiker.

Von dieser pädagogischen Vision träumte man nicht nur in der Bundesrepublik Deutschland, sondern auch in Großbritannien, Skandinavien, den Vereinigten Staaten von Amerika und in vielen anderen Ländern. In den USA radikalisierte Benjamin Bloom, ein sehr angesehener und einflussreicher Bildungsforscher, die wissenschaftlich höchst spekulative, aber pädagogisch für gültig gehaltene Annahme: „Was irgendeine Person in der Welt lernen kann, kann fast jede Person lernen, vorausgesetzt, dass das frühere und gegenwärtige Lernen unter angemessenen Bedingungen erfolgt (...) die Theorie bietet eine optimistische Perspektive auf das, was Bildung für Menschen leisten kann« (Bloom 1976, 7).

Was für eine pädagogische Utopie; aber auch welch ein psychologisches Fehlurteil! Theoretische Grundlage der pädagogischen Verheißung Blooms war die schlichte, in den 60er Jahren systematisch untersuchte Tatsache eines kompensatorischen Verhältnisses zwischen dem individuellen Niveau der Lernvoraussetzungen und der benötigten Lernzeit zur Erreichung eines anspruchsvollen Bildungszieles. Wer weniger kann und weniger weiß als andere, muss eben mehr Zeit für zusätzliche, nachholende und ergänzende Lernschritte investieren, um jene Aufgaben meistern zu können, die bessere Schüler bereits früher beherrschen. Man spricht deshalb vom Modell des zielerreichenden Lernens und Lehrens, das aber erst in Verbindung mit der theoretischen Annahme praktischen Sinn

${ }^{1}$ Wiederabdruck mit freundlicher Genehmigung von Bildung und Begabung gGmbH und Familie Weinert; aus Wagner, H. (Hrsg.) (2000): Begabung und Leistung in der Schule. Modelle der Begabtenförderung in Theorie und Praxis. 2. überarbeitete und erweiterte Auflage. Bad Honnef: Karl Heinrich Bock. Festvortrag anlässlich der Hauptversammlung der Max-PlanckGesellschaft in Dortmund am 11. Juni 1999. 
macht, dass nämlich die zusätzlich benötigte Lernzeit als Funktion des vorhergehenden systematischen Lernens kontinuierlich abnimmt. Diese Erwartung konnte empirisch nicht bestätigt werden! Ein statistischer Witzbold hatte deshalb schon früh errechnet, dass man die Schulzeit einfach auf einhundertzwanzig Jahre verlängern müsste, damit jeder und jede Heranwachsende einen universitären Abschluss erreicht.

Aus den umfangreichen und vielfältigen seriösen Forschungsbemühungen zu dieser Thematik mussten die Verfechter einer pädagogischen Egalisierungsillusion mehr oder minder resignativ oder aggressiv zur Kenntnis nehmen, dass das Modell des zielerreichenden Lernens zwar für umschriebene Bildungsziele - z. B. beim Erwerb des Lesens, des Schreibens, oder der Arithmetik - brauchbar und sogar notwendig ist, dass aber auch unter optimalen schulischen Bedingungen gilt: Hält man bei unterschiedlich begabten Schülern die Lernzeit konstant, so ergeben sich große Leistungsunterschiede; will man die gleichen anspruchsvollen Schulleistungen erreichen, so sind - wenn es überhaupt gelingt - extreme Differenzen der Lernzeit zu erwarten (Slavin 1987).

Unerfüllbare ideologisch-theoretische Hoffnungen sind Wurzeln vieler praktischer Enttäuschungen, was zumindest in den Sozialwissenschaften häufig zu ebenso ideologieanfälligen Kontrasttheorien führt. So auch im Bereich von Begabung und Lernen, also bei der klassischen Kontroverse über die Erb- oder Umweltdeterminiertheit geistiger Leistungen und Leistungsunterschiede. Innerhalb weniger Jahre schlug das Pendel von der dominierenden Wertschätzung des Lernens zur bevorzugten Fixierung auf stabile Begabungsunterschiede zurück. Dieser Trend ist weltweit zu beobachten, am radikalsten hat er sich in der Studie von Herrnstein und Murray »The Bell Curve» (1994) artikuliert.

Das dickleibige, mit Statistiken überladene Werk enthält zwei grundlegende wissenschaftliche Annahmen und eine daraus nicht ableitbare provokante gesellschaftspolitische Schlussfolgerung:

a) Das Zusammenspiel von Unterschieden der genetischen Ausstattung und der sozioökonomischen Lage in den USA führt in der Entwicklung von Kindern schon sehr früh zu stabilen interindividuellen Unterschieden in kognitiven Kompetenzen, motivationalen Tendenzen und sozialen Verhaltensmustern. Dabei kommt dem Intelligenzquotienten für die gesamte Lebensführung eine Schlüsselrolle zu.

b) Sozialpädagogische, schulorganisatorische und didaktische Interventionen führen zu keiner bedeutsamen Reduzierung der Intelligenzunterschiede zwischen verschiedenen Kindern und vor allem nicht zu einer Anhebung niedriger intellektueller Fähigkeiten. »Zusammenfassend lässt sich sagen, dass alle Versuche zur Steigerung der Intelligenz eine Geschichte bilden, die durch große Hoffnungen, überzogene Behauptungen und enttäuschende Ergebnisse charakterisiert ist« (Herrnstein/Murray 1994, 389).

c) Nach Meinung von Herrnstein und Murray sollte man deshalb künftig auf kompensatorische Förderprogramme, auf Maßnahmen zur Reduzierung kognitiver, schulischer und beruflicher Ungleichheiten sowie auf die öffentliche Propagierung von Chancengleichheit als eines gesellschaftlichen Wertes verzichten und die frei werdenden finanziellen Ressourcen in die Bildung derjenigen Jugendlichen investieren, die über ein großes geistiges Potential verfügen und die später den größten Teil des nationalen Sozialprodukts erwirtschaften. Im Übrigen müsse die amerikanische Gesellschaft wieder lernen, mit individuellen und sozialen Ungleichheiten in Würde und gesellschaftlicher Harmonie zu leben.

Der daraufhin ausbrechende differentialpsychologische, schulpädagogische und gesellschaftspolitische Streit wird inzwischen durchweg als »Krieg« bezeichnet, der - fünf Jahre nach Erscheinen des Buches - weniger zur Klärung der Sachfragen als zur Bildung sozialanthropologischer Profilierungsneurosen beigetragen hat.

Bei den zwei wissenschaftlich unhaltbaren Thesen von Bloom einerseits und von Herrnstein und Murray andererseits handelt es sich selbstverständlich um Extrempositionen. Eine Durchsicht der wissenschaftlichen wie der populärwissenschaftlichen Literatur zeigt aber, dass sich die radikalen Standpunkte in vielfältigen Schattierungen als mehr oder minder einseitige theoretische Voreingenommenheiten in vielen humanbiologischen und pädagogisch-psychologischen Arbeiten wiederfinden.

Was an der zeitgeschichtlich interessanten Veränderung von stark umweltsoziologischen Einstellungen zu betont 
erbtheoretischen Positionen innerhalb weniger Jahre besonders erstaunt, ist das Fehlen neuer Erkenntnisse, die als wissenschaftliche Erklärungen dafür dienen könnten. Auf dem Gebiet der verhaltens- wie der molekulargenetischen Intelligenzforschung gibt es zwar viele und große methodische Fortschritte, aber keine revolutionären empirischen Befunde, die einen radikalen Wechsel wissenschaftlicher Annahmen über die Notwendigkeiten, Möglichkeiten, Grenzen und Spielräume der Bildung für die geistige Entwicklung des Menschen rechtfertigen würden.

- Dass 50 Prozent oder etwas mehr der interindividuellen Fähigkeitsunterschiede durch Erbeinflüsse erklärbar sind, weiß man aus den übereinstimmenden Resultaten von Zwillingsuntersuchungen und Adoptionsstudien seit langem.

- Dass die Einflüsse kollektiv erfahrener Umwelten im Verlauf des Lebens ab- und die Wirkungen individuell erlebter Eigenwelten zunehmen, ist ebenfalls nicht neu.

- Dass Erb- und Umwelteffekte nicht unabhängig voneinander sind, sondern in starkem Maße kovariieren, liegt seit Jahrzehnten auf der Hand (vgl. Sternberg/ Grigorenko 1997).

Das Fatale am gegenwärtigen Erkenntnisstand ist, dass sowohl erb- als auch umwelttheoretische Deutungen der individuellen Entwicklung möglich sind. Daraus ergibt sich eine erhebliche Ideologieanfälligkeit der gesamten Forschungsrichtung. Denn die Tatsache, »dass ungefähr die Hälfte der Varianz intellektueller Leistungen genetischen Differenzen zwischen den Individuen zuzuschreiben ist «, bedeutet trivialerweise auch, »dass ungefähr die Hälfte der Varianz in ihrem Ursprung nicht genetisch determiniert ist« (Plomin 1988, 9).

Auch wenn man in naher Zukunft massive molekulargenetische Erkenntnisfortschritte erwarten kann, dürfte sich an der pädagogisch relevanten wissenschaftlichen Gesamtsituation nach der Überzeugung führender Fachvertreter auf diesem Forschungsgebiet wenig ändern. Es erscheint deshalb unergiebig, wieder und wieder nach abschließenden wissenschaftlichen Antworten auf die fundamentale Frage nach den biologischen Erb- und soziokulturellen Umweltdeterminanten der geistigen Entwicklung zu suchen. Zweckmäßiger dürfte es sein, nach der Stabilität kognitiver Leistungsunterschiede zu verschiedenen Zeitpunkten des menschlichen Lebenslaufes zu fahnden, um die Möglichkeiten, Bedingungen, aber auch Grenzen der Veränderbarkeit interindividueller Leistungsdifferenzen zu analysieren.

Das soll im Folgenden geschehen. Dabei wird die einschlägige Literatur vorwiegend als Hintergrundinformation benutzt, um einige Ergebnisse aus drei größeren Studien zu interpretieren, die in den zwei vergangenen Jahrzehnten am Max-Planck-Institut für psychologische Forschung in München durchgeführt wurden:

a) Eine Longitudinalstudie zur Genese individueller Kompetenzen (kurz: LOGIK), an der mehr als 220 Kinder zwischen ihrem 3. und 12. Lebensjahr teilnahmen und mehrmals jährlich beobachtet, befragt, getestet und unter experimentellen Bedingungen studiert wurden. Nach 5-jähriger Unterbrechung konnten 94 Prozent der inzwischen 17 Jahre alten Jugendlichen für eine erneute intensive Untersuchung gewonnen werden, so dass nunmehr Längsschnittdaten für einen Zeitraum von vierzehn Jahren verfügbar sind (Weinert/Schneider 1999).

b) Eine Untersuchung über schulorganisierte Lernangebote zur Sozialisation von Talenten, Interessen und Kompetenzen (kurz: SCHOLASTIK), an der sich neben 118 Kindern der LOGIK-Stichprobe mehr als 1100 Mitschüler in 54 Klassen während der gesamten Grundschulzeit beteiligten. Die Kinder wurden pro Schuljahr neun Mal im regulären Unterricht beobachtet und getestet (Weinert/Helmke 1997). Abbildung 1 gibt einen Überblick über den Verlauf und die Verzahnung der kombinierten LOGIK- und SCHOLASTIK-Studien.

c) Eine genetisch orientierte Lebenslaufstudie zur Differentialentwicklung (kurz: GOLD) mit ein- und zweieiigen Zwillingen. Begonnen wurde diese Untersuchung im Jahr 1937 unter Leitung von Kurt Gottschaldt am Kaiser-Wilhelm-Institut für Anthropologie, menschliche Erblehre und Eugenik. Beteiligt waren 90 Zwillingspaare im Alter von 11 Jahren. 53 der Paare wurden 1965/1966, also im 40. Lebensjahr, erneut untersucht. Nach dem Tode von Kurt Gottschaldt konnte die Studie seit 1992 am Max-Planck-Institut für psychologische Forschung weitergeführt werden. Neben den verbliebenen - inzwischen 70 Jahre alten - 32 Zwillingspaaren der Gottschaldt'schen Längsschnittstichprobe wurden zusätzlich mehr als 180 Zwillingspaare im Alter von 65-85 Jahren für eine Teilnahme an der Studie gewonnen 


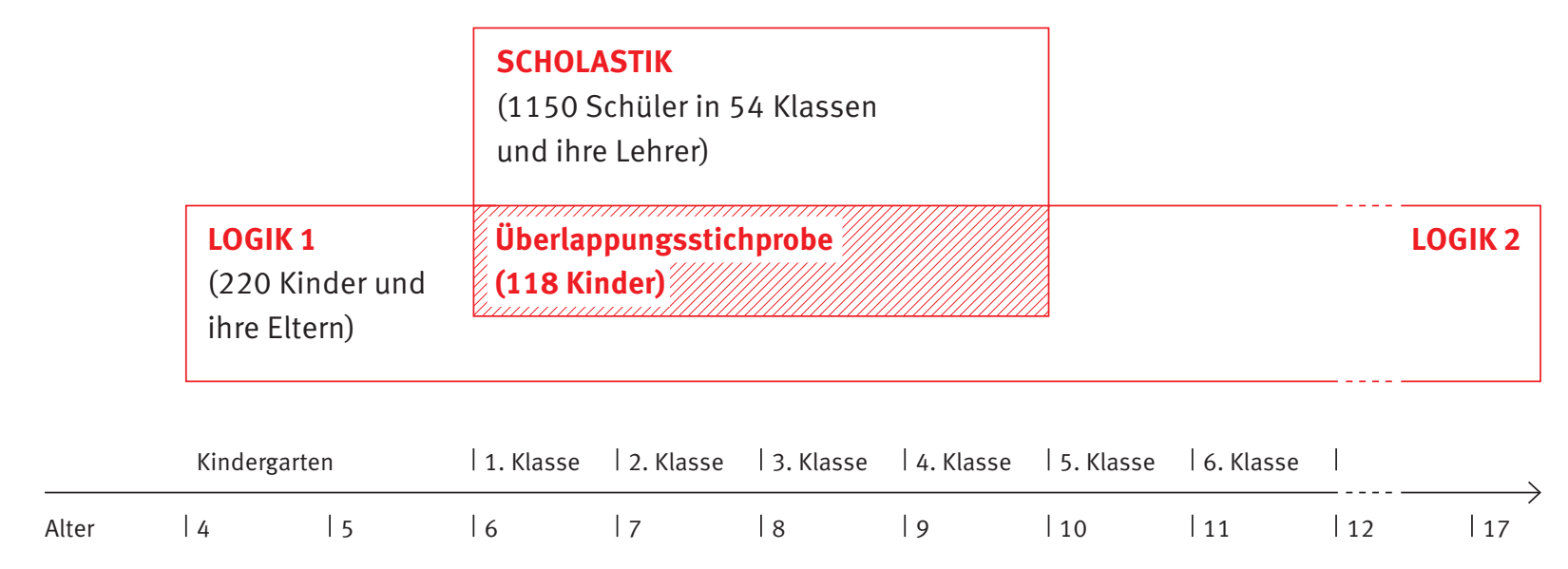

Abb. 1: Verzahnung der beiden Projekte LOGIK und SCHOLASTIK.

(Weinert/Geppert 1998). Abbildung 2 enthält die Details der GOLD-Untersuchung.

Mit Hilfe der drei Datensätze, die in dieser Form nur unter den besonderen, forschungsförderlichen Bedingungen eines Max-Planck-Instituts gewonnen werden konnten, lassen sich einige wesentliche Fragen zur differentiellen Entwicklung geistiger Kompetenzen während der Lebensspanne beantworten. Das wird im Folgenden in fünf kurzen Schritten geschehen:

1. Individuelles Lernen und der Erwerb geistiger Kompetenzen

2. Interindividuelle Unterschiede beim Erwerb geistiger Kompetenzen

3. Die Stabilität von individuellen Intelligenz-, Lern- und Leistungsunterschieden

4. Die Rolle der Schule beim Erwerb geistiger Kompetenzen

5. Determinanten der beruflichen Entwicklung.

\section{Die Bedeutung des individuellen Lernens für den Erwerb} geistiger Kompetenzen

Welche Rolle spielen kognitive Lernprozesse bei der Entwicklung geistiger Fähigkeiten und Kompetenzen? Verweisen die Besonderheiten des frühen Spracherwerbs, die universellen Regularitäten der psycho-motorischen Entwicklung oder die alterstypischen Veränderungen des kindlichen Denkens nicht auf genetisch vorprogrammierte hirnorganische Reifungsprozesse, so dass dem Lernen nur eine marginale verhaltensspezifizierende Bedeutung zu- kommt? Dem ist nicht so! Alle geistigen Kompetenzen müssen gelernt werden. Lernen ist in der Ontogenese ein ubiquitäres Geschehen, dessen Ergebnisse wir im Verhalten und Erkennen als Entwicklungskurven, Wachstumsfunktionen oder Veränderungssequenzen darstellen können. Vorausgesetzt, man verfügt über zuverlässig standardisierte Messverfahren, die weder alterstypische Bodennoch Deckeneffekte aufweisen, so lassen sich im Kindesund Jugendalter in allen Verhaltensbereichen massive Leistungsverbesserungen nachweisen.

Also ließe sich die geistige Entwicklung einfach mit kognitivem Lernen gleichsetzen? Das ist einer der gravierenden Fehlschlüsse mancher Lerntheoretiker. Lernen bedeutet nämlich vor allem in der frühen Kindheit nicht das Erwerben beliebiger Informationen und Verhaltensdispositionen zu beliebigen Zeitpunkten, sondern unterliegt einigen grundlegenden Beschränkungen.

Der Mensch ist zum Zeitpunkt der Geburt keineswegs eine Tabula rasa, sondern verfügt neben den generellen assoziativen Lernmechanismen über domänspezifische Lernpotentiale, die sich bereits im Säuglingsalter nachweisen lassen. Dazu gehören spezielle Formen der sozialen Wahrnehmung, linguistische und numerische Kompetenzen sowie »Einsichten « in physikalische und biologischpsychologische Prinzipien. Diese artspezifische geistige Grundausstattung ist im strengen Sinn des Wortes das Erbe unserer Stammesgeschichte. Mit anderen Worten: Es gibt schon in der frühesten Kindheit domänspezifische Lernpotentiale, die man sich als privilegierte hirnorga- 


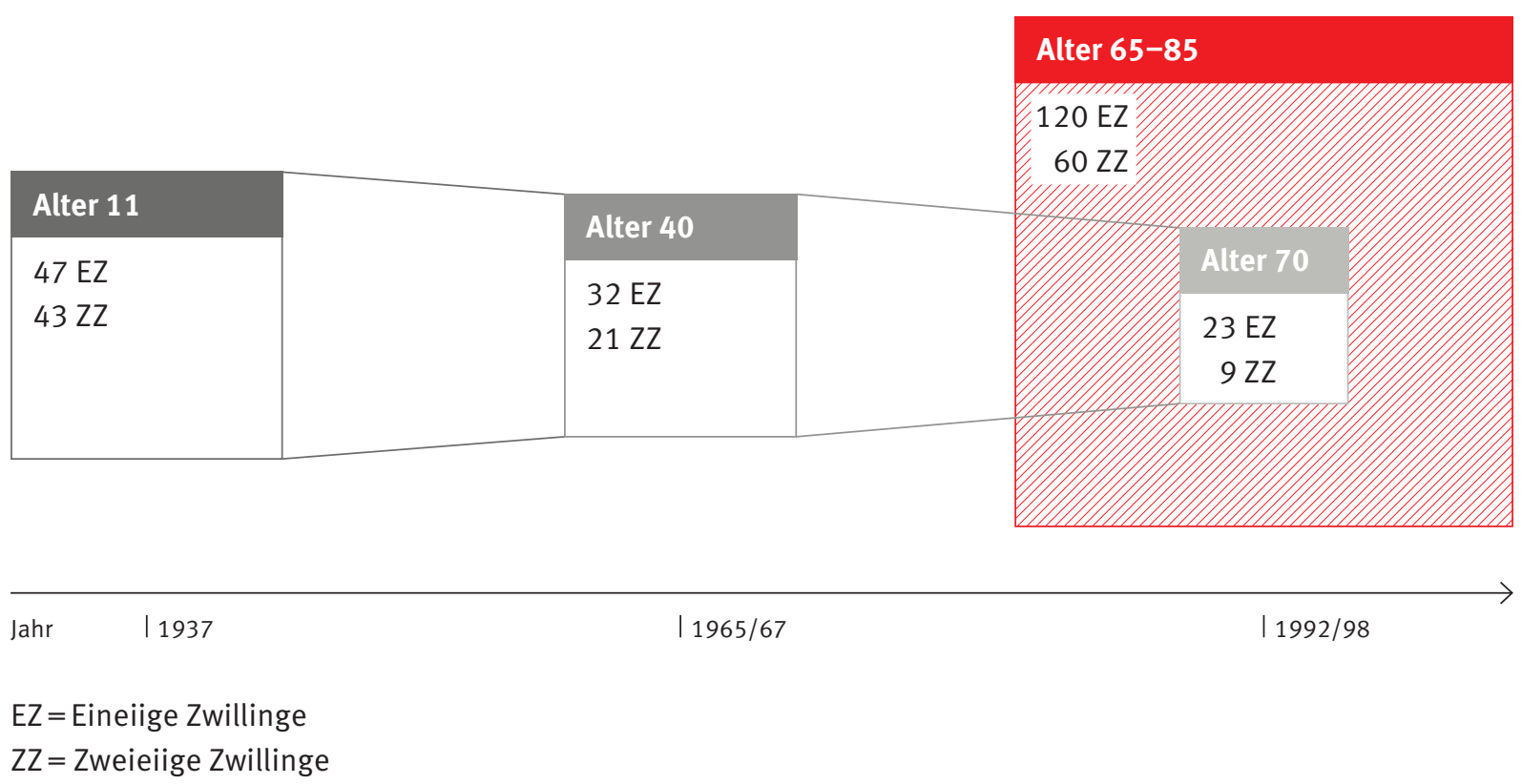

Abb. 2: Verlauf der GOLD-Studie.

nische Verschaltungsmöglichkeiten vorstellen kann, und die auf der einen Seite die Spielräume möglicher Erfahrung einengen, auf der anderen Seite aber gerade deshalb die Wirksamkeit bestimmter Lernvorgänge dramatisch verstärken. Diese Lernprozesse sind kumulativ, also aufeinander aufbauend, folgen einer mehr oder minder deutlichen Sachlogik und führen zu deklarativen wie prozeduralen Kompetenzen, die sich nicht als Bündel gespeicherter Informationen, sondern als organisierte Wissenssysteme charakterisieren lassen.

Sinnvolles Lernen heißt demnach von Anfang an, neue Informationen in eine bereits verfügbare Wissensbasis einzugliedern; Verstehen ergibt sich aus dem Zusammenspiel wohlorganisierter Informationssysteme, die wir als Wissen bezeichnen. Es ist deshalb mehr als ein metaphorisches Sprachspiel, wenn man das Wissen auch schon bei kleinen Kindern in Analogie zu wissenschaftlichen Theorien betrachtet, denn es erlaubt den Heranwachsenden auf allen Altersstufen, ihre Welt auf ihre Weise zu verstehen, Ereignisse plausibel zu erklären, Dinge vorherzusagen und dem eigenen Handeln Orientierung zu geben.

\section{Interindividuelle Unterschiede beim Erwerb geistiger Kompetenzen}

Neben den entwicklungspsychologischen und sachlogischen Beschränkungen des Lernens gibt es differentialpsychologische Begünstigungen und Benachteiligungen, deren wissenschaftliche Erfassung im Säuglings- und frühen Kindesalter noch ganz am Anfang steht.

Konzentriert man sich auf die kognitiven Veränderungen während der Vorschul- und Schulzeit, so erweist sich das Entwicklungstempo als ein überaus interessantes differentielles Merkmal. Kinder unterscheiden sich schon früh in der Geschwindigkeit, Menge und Qualität von Lernprozessen, die in einer bestimmten Zeiteinheit stattfinden. Daraus ergeben sich individuell unterschiedliche Wachstumskurven. Abbildung 3 zeigt den erwarteten Verlauf. Von besonderer theoretischer Bedeutung ist dabei, dass die Steigungswinkel der Kurven zu verschiedenen Zeitpunkten der Entwicklung eine brauchbare Prognose der Asymptote, also des erreichbaren Entwicklungsniveaus erlauben. 


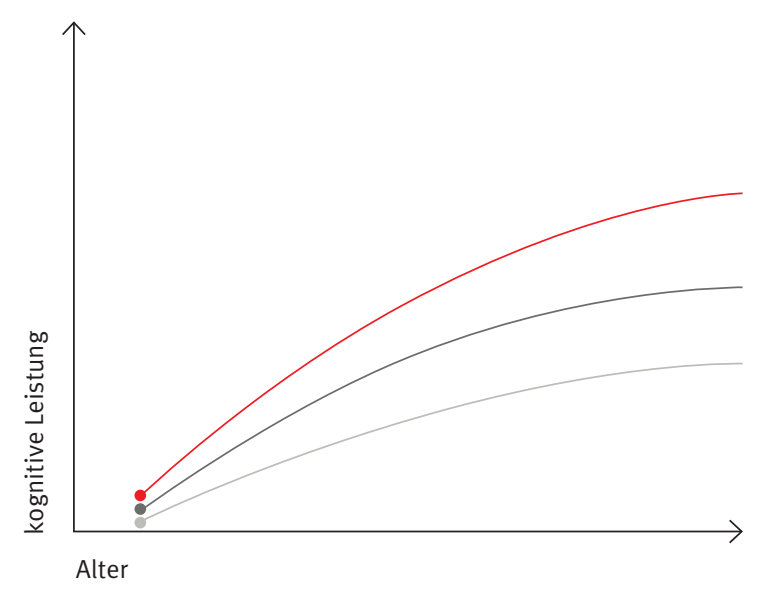

Abb. 3: Individuelle Unterschiede in der Entwicklung kognitiver Leistungen (beobachtete Entwicklungsverläufe).

Im Vergleich zu den in Abbildung 3 dargestellten naturwüchsigen Entwicklungskurven halten es optimistische Bildungstheoretiker allerdings für möglich, dass sich die vorfindbaren interindividuellen Entwicklungsunterschiede durch Optimierung der schulischen Lernprozesse angleichen lassen. Abbildung 4 veranschaulicht dieses Entwicklungsmodell.

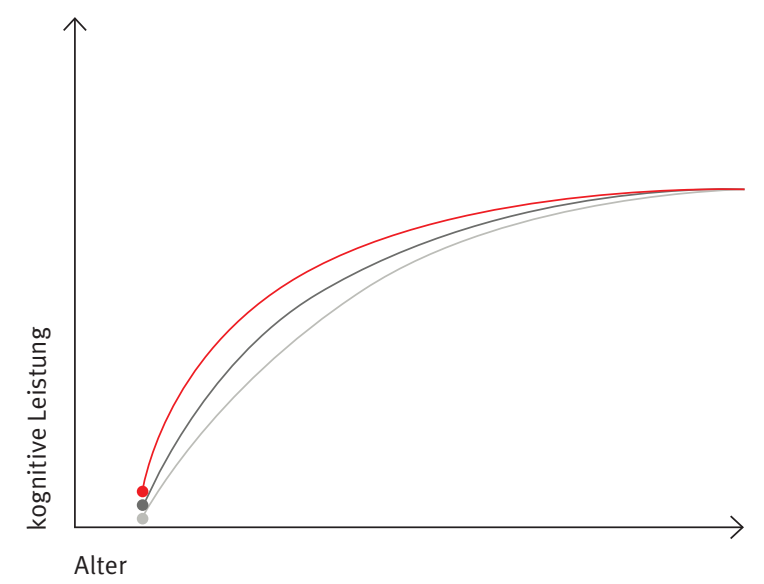

Abb. 4: Individuelle Unterschiede in der Entwicklung kognitiver Leistungen (unter optimalen Lernbedingungen erhoffte Entwicklungsverläufe).

Betrachtet man die zu dieser Kontroverse vorliegenden empirischen Befunde, so belegen sie eindeutig die Stabilität interindividueller Unterschiede bei der Entwicklung anspruchsvoller geistiger Leistungsfähigkeiten. Nimmt man als Beispiel die Entwicklung der Intelligenz, so zeigt Abbildung5, dass sich die drei mit Hilfe einer Clusteranalyse identifizierten Niveaugruppen parallel mit einer leichten Spreizungstendenz verändern.

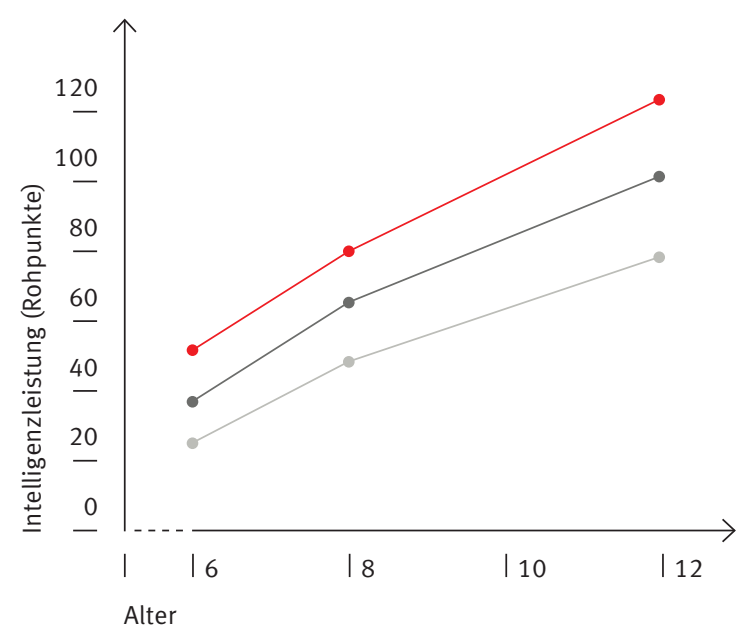

- gutes Leistungsniveau

- durchschnittliches Leistungsniveau

- schlechtes Leistungsniveau

Abb. 5: Entwicklung der Intelligenzleistungen bei schlechtem, durchschnittlichem und gutem Leistungsniveau.

Dieses Muster der differentiellen Entwicklung scheint für das gesamte Kindesalter typisch zu sein. Es muss allerdings in dreifacher Hinsicht spezifiziert werden:

a) Bei allen kognitiven Kompetenzen, bei denen sich in der Frühphase der Ontogenese bedeutsame interindividuelle Unterschiede zeigen, bleiben diese Differenzen langfristig auch unter dem Einfluss extern angeregter und geförderter Lernprozesse erhalten und beeinflussen in bedeutsamer Weise das individuell erreichbare, maximale Leistungsniveau im frühen Erwachsenenalter.

b) Bei den meisten kognitiven Kompetenzen können fast alle geistig gesunden Kinder ein Entwicklungsniveau erreichen, das ihre spätere aktive Teilhabe am sozialen, kulturellen und beruflichen Leben der Gesellschaft ermöglicht. Das erfordert allerdings eine Vielzahl und Vielfalt von Lernprozessen, die vor allem bei den leistungsschwächeren Kindern möglichst optimal gefördert werden müssen. 
c) Für die Entwicklung vieler geistiger Kompetenzen gilt unter den gegebenen sozio-kulturellen Bedingungen sogar eine Art Matthäusprinzip. Wer zu einem bestimmten Zeitpunkt im Vergleich zu anderen über bessere individuelle Lernvoraussetzungen verfügt, wird von gleichen Lernangeboten, Lerngelegenheiten und Lernanforderungen stärker profitieren. Das führt in der kognitiven Entwicklung notwendigerweise zu Schereneffekten, das heißt zu einer Spreizung der interindividuellen Leistungsunterschiede, wenn nicht kompensatorische Einflüsse wirksam werden. Das ist zum Beispiel in den deutschen Grundschulen programmatisch der Fall, sollte aber auf den Erwerb aller basalen Leistungsdispositionen ausgeweitet werden, wobei den Hauptschulen eine besonders wichtige Funktion zukommt.

\section{Die Stabilität interindividueller Intelligenz-, Lern- und Leistungsunterschiede}

Versteht man unter Begabung die Gesamtheit jener kognitiven Faktoren, welche die Stabilität von individuellen Lern- und Leistungsunterschieden auch unter variablen Umwelteinflüssen und unter gezielten didaktischen Interventionen determinieren, so stößt man einerseits auf relativ allgemeine intellektuelle Fähigkeiten und andererseits auf domänspezifische Kompetenzen. Abbildung 6 veranschaulicht beispielhaft die Zusammenhänge innerhalb und zwischen der Entwicklung intellektueller Fähigkeiten und mathematischer Kompetenzen während der Vor- und Grundschulzeit.

Bei den in dieser Grafik benutzten Koeffizienten bedeutet der Wert +1 die maximale Gleichheit der Unterschiede, der Wert 0 verweist auf einen fehlenden Zusammenhang. Alle Daten stammen von denselben Kindern der LOGIKStudie. Analysiert man die im Bild dargestellten zwei Stränge der kognitiven Entwicklung, so beeindruckt vor allem die zunehmende Stabilität der Kompetenzunterschiede zwischen verschiedenen Kindern. Das gilt sowohl für die allgemeine Intelligenz als auch für die mathematische Kompetenz. Wer am Anfang der Grundschule besser ist als andere, besitzt eine überzufällige Wahrscheinlichkeit, dass er oder sie eine ähnliche Position auch am Ende der Grundschulzeit einnimmt. Leider gilt die gleiche Stabilität auch für die weniger leistungstüchtigen Schüler. Dieser Trend nimmt im Verlauf der späteren Kindheit und des Jugendalters zu, - sieht man von gelegentlichen Leistungsirritationen bei manchen Heranwachsenden $a b$.

Es wäre jedoch eine einseitige Interpretation der empirischen Daten, würde man nicht auch die Variabilität der kognitiven Entwicklung hervorheben. Einige Kinder verbessern, andere verschlechtern sich im Vergleich zu ihren Mitschülern. Das gilt sowohl für die intellektuellen Fähigkeiten als auch für die verschiedenen Schulleistungen. Drastische Veränderungen sind allerdings seltene Ausnah-

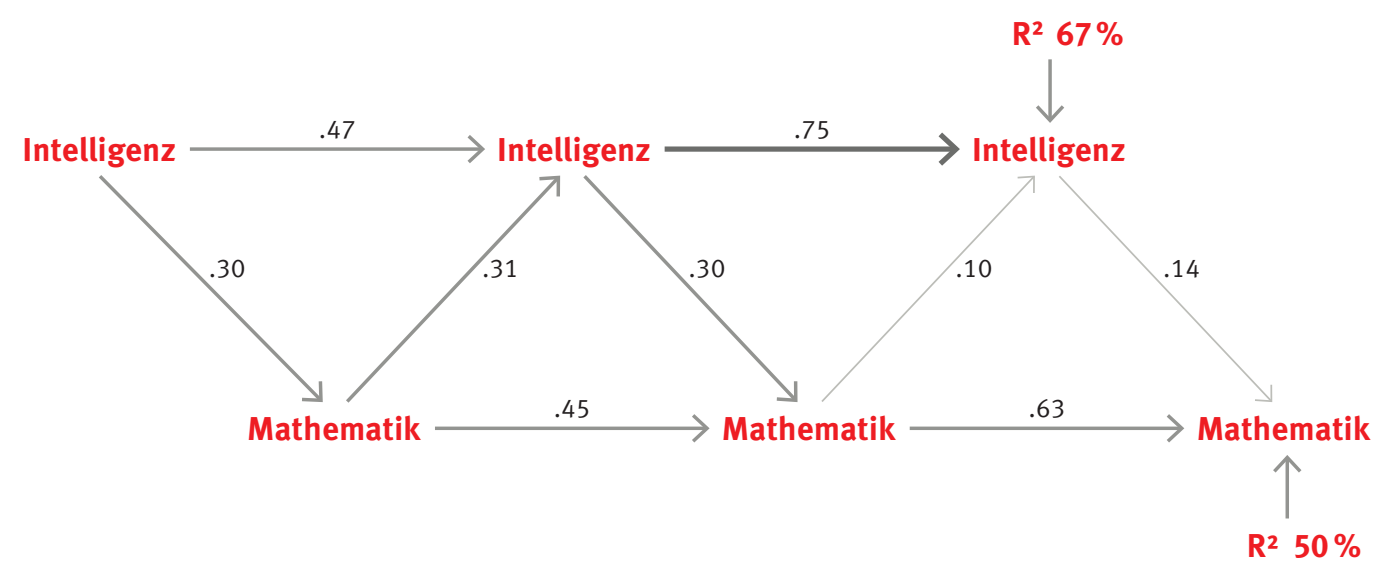

Kindergarten

|1. $\quad$ | 2.

Grundschule

| 2. J. Jahr

I.

$\mid 2$.

13.

| 4. Klasse 
men. Leider ist es in den bisherigen Datenanalysen noch nicht gelungen, die Stabilität und Variabilität der interindividuellen Entwicklungsunterschiede als Folge interner Personmerkmale und/oder externer Umwelteinflüsse zu erklären.

Von erheblichem wissenschaftlichem wie praktischem Interesse ist die Tatsache, dass auch bedeutsame individuelle Differenzen in den mathematischen Kompetenzen schon im Vorschulalter nachweisbar sind und die weitere Entwicklung in diesem Bereich stärker determinieren als die allgemeinen Intelligenzunterschiede.

Dabei gilt: Kompetenz ist nicht gleich Kompetenz, Wissen ist nicht gleich Wissen. Überdurchschnittliche intellektuelle Fähigkeiten, spezielle Begabungen und verständnisintensive Lernprozesse erhöhen die Qualität des erworbenen Wissens und Könnens, das heißt dessen Organisiertheit, Abstraktheit, Anpassungsfähigkeit und Zugriffsmöglichkeit. Man kann deshalb von der Entwicklung mehr oder minder intelligenten Wissens sprechen.

Am offenkundigsten ist die Stabilität der individuellen Merkmalsunterschiede bei den intellektuellen Fähigkeiten. Abbildung 6 zeigt diesen Trend für die Zeit der Kindheit. Die gleiche Tendenz setzt sich im Jugend- und frühen Erwachsenenalter fort, wie die Ergebnisse zahlreicher Längsschnittstudien übereinstimmend belegen. Überaus beeindruckend sind die extrem geringen interindividuellen Veränderungen zwischen dem 40. und 70. Lebensjahr, wie sich aus Abbildung 7 ersehen lässt.

Ordnet man die Probanden aufgrund der 1965 erzielten Testleistungen in eine Rangreihe, so ergeben sich über eine dreißigjährige Zeitspanne hinweg fast keine Positionsverschiebungen, obwohl auch in dieser Altersperiode vielfältige Prozesse des Lernens, des Wissenserwerbs und der Gewinnung neuer Erfahrungen stattfinden.

Alles in allem: Kinder werden älter und allmählich erwachsen, erwerben dabei große Mengen von Informationen, verbessern ihre geistigen Kompetenzen in markanter Weise, erlernen verschiedenste Berufe und bleiben im Vergleich zu ihren Altersgenossen im intellektuellen Niveau doch fast die alten.

Was sind die Ursachen dieser Stabilität kognitiver Merkmalsdifferenzen? Natürlich muss bei der Beantwortung

\begin{tabular}{lll}
$\begin{array}{l}\text { HAWIE- } \\
\text { Kennwert }\end{array}$ & $\begin{array}{l}\text { Stabilität } \\
(40 .-70 . \text { Lebensjahr })\end{array}$ & $\begin{array}{l}\text { Korrigierte } \\
\text { Stabilität }\end{array}$ \\
\hline Gesamt-IQ & .88 & .92 \\
\hline Verbal-IQ & .88 & .92 \\
\hline Handlungs-IQ & .79 & .93 \\
\hline
\end{tabular}

Abb. 7: Stabilität der Intelligenzkennwerte zwischen dem 40. und 70. Lebensjahr, korrigiert durch die Reliabilität der Messinstrumente.

dieser Frage an erster Stelle die Verschiedenheit der genetischen Ausstattung genannt werden (vgl. Abb. 8).

Abbildung 8 zeigt einen repräsentativen Überblick über die Befunde zur Ähnlichkeit des Intelligenzquotienten bei genetisch identischen (erste zwei Zeilen), genetisch verwandten (mittlere vier Zeilen) und genetisch nicht verwandten Menschen (letzte zwei Zeilen), die entweder zusammen oder getrennt leben bzw. aufgewachsen sind. Die Graphik veranschaulicht die etwas größere Bedeutung der Erb- im Vergleich zu den Umwelteinflüssen auf die intellektuelle Entwicklung. Die für ein- und zweieiige

Bedingungen $r$

\section{Genetisch identisch}

EZ/zus. aufgewachsen

EZ/getr. aufgewachsen

\section{Genetisch verwandt}

ZZ/zus. aufgewachsen Geschwister/zus. aufgewachsen

Eltern-Kind/zus. lebend

Eltern-Kind/getr. durch Adpotion

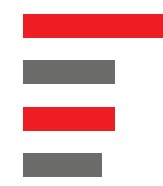

\section{Genetisch nicht verwandt}

Nicht verwandte Kinder/

zus. aufgewachsen

Adoptiveltern-Adoptivkind

Abb. 8: Korrelationskoeffizienten für die IQ-Werte von genetisch identischen, genetisch verwandten und genetisch nicht verwandten Menschen. 
Zwillinge dargestellten Werte stimmen mit den entsprechenden Ergebnissen der GOLD-Studie überein. Bei 65bis 70-jährigen eineiigen Zwillingen sind die IQ-Differenzen extrem gering. Sie liegen fast alle innerhalb einer halben Standardabweichung, um es technisch auszudrücken (Dörfert 1996).

Neben der genetischen Ausstattung lässt sich die hohe Stabilität individueller Intelligenzunterschiede aber natürlich auch auf Umwelteinflüsse zurückführen, die bereits in der Kindheit bedeutsam mit dem Genotypus korrespondieren. Das bedeutet in den meisten Fällen nicht nur die Identität der leiblichen Eltern als Träger des genetischen wie des sozio-kulturellen Erbes, sondern auch die mit dem Alter zunehmende Selektion und Gestaltung der individuellen Umwelt durch den einzelnen Menschen, - selbstverständlich stets in mehr oder minder großer Übereinstimmung mit dem Genotypus.

Diesen mächtigen kausalgenetischen Bedingungen für die Entstehung, Aufrechterhaltung und Verstärkung der Stabilitäten interindividueller Unterschiede in den kognitiven Kompetenzen stehen nur begrenzte kompensatorisch oder gegenläufig wirkende Mechanismen gegenüber. Es können dies die idiosynkratischen sozialen Interaktionen innerhalb der Familie, die gleichaltrigen Freunde und die durch sie definierten neuen sozialen Bezugsgruppen, vielfältige Medieneinflüsse, Schulen, berufliche Konstellationen, Eheschließungen, kritische Lebensereignisse und Zufälle der verschiedensten Art sein, wobei die Variabilität der individuellen Lebensverhältnisse in der Regel sehr begrenzt ist.

Die Entwicklung theoretischer Modelle zur Verknüpfung all dieser Einflussfaktoren auf die Stabilität und Variabilität interindividueller Unterschiede der geistigen Entwicklung ist ein wissenschaftliches Feld, in dem es mehr offene Fragen als befriedigende theoretische Antworten gibt. Was hier aus guten Gründen unterbleibt, ist der beliebte Versuch, die Erbeinflüsse, die Effekte der von Zwillingen gemeinsam erlebten Umwelt und die Wirkungen der von jedem Individuum spezifisch erfahrenen Umwelt auf die intellektuelle Entwicklung zu separieren und in Prozentanteilen auszudrücken. Das kommt nämlich in mancher Hinsicht dem Unterfangen gleich, eine Gemüsesuppe erb- und umweltspezifisch analysieren zu wollen, wie der »New Yorker« vor einiger Zeit sarkastisch bemerkte.

\section{Die Rolle der Schule beim Erwerb geistiger Kompetenzen}

Weltweit ist zum gegenwärtigen Zeitpunkt niemand in der Lage, die Zusammenhänge zwischen individuellen Lernvoraussetzungen, typischen Lernprozessen und stabilen Leistungsunterschieden durch Erb-, Umwelt-, Entwicklungs- und Situationseinflüsse befriedigend zu erklären. Die wissenschaftlich zweifelsfrei nachgewiesenen Unterschiede in der Lernwirksamkeit und - damit zusammenhängend - im Leistungsniveau zwischen verschiedenen Kindern rechtfertigen aber die theoretische Annahme von stabilen interindividuellen Begabungsdifferenzen. Welche Rolle spielen diese im Bildungssystem? Um auf diese Frage eine Antwort geben zu können, muss man sie zuerst in zwei sehr unterschiedliche Teilfragen zerlegen, denn es geht zum einen um die Lernfortschritte aller, also um das Erreichen bestimmter Bildungsziele; zum anderen handelt es sich um die Reduzierung von Leistungs- und Begabungsunterschieden zwischen den Individuen.

Für den Erwerb geistiger Kompetenzen, insbesondere jener, die wir in unserer wissenschaftlich-technisch überformten Welt benötigen, ohne dass sie in der naturwüchsigen Umwelt der Kinder "gelehrt« werden, sind Schulen notwendige, unentbehrliche und auch wirksame Institutionen. Die nachwachsende Generation lernt im Wesentlichen das, was ihr an Lerninhalten in den Schulen angeboten wird, und sie lernt es - allerdings begabungsabhängig - in einer Güte, die weitgehend durch die Qualität des Unterrichts beeinflusst wird.

Die Situation stellt sich völlig anders dar, wenn es nicht um Lernfortschritte und Leistungssteigerungen bei allen Schülern geht, sondern um den Abbau von Leistungsdifferenzen und Begabungsunterschieden zwischen verschiedenen Schülern. Das ist nur in sehr begrenztem Maße möglich.

Aus diesen gut gesicherten wissenschaftlichen Befunden lassen sich zwei divergierende schulpraktische Schlussfolgerungen ziehen:

a) Die optimistische Botschaft bezieht sich auf das Lernen als dem wichtigsten Mechanismus der kognitiven Entwicklung. Die individuellen Lernpotentiale werden in unseren Schulen noch keineswegs optimal ausgeschöpft. Das gilt sowohl für Hochbegabte als auch für begabungsschwächere Schüler, die eine intensive 
didaktische Förderung benötigen. Noch so gut gemeinte sozialpädagogische Maßnahmen können dafür kein Ersatz, sondern nur eine Ergänzung sein.

b) Die pessimistische wissenschaftliche Botschaft gilt dem Versuch, die stabilen Begabungs-, Lern- und Leistungsunterschiede zwischen den Schülern aufzuheben. Sowohl in der Münchner Hauptschulstudie als auch in der SCHOLASTIK-Untersuchung konnte überzeugend nachgewiesen werden, dass massive Bemühungen in dieser Richtung sogar dysfunktional für die individuellen Lernfortschritte sein können.

\section{Persönliche Determinanten der beruflichen Entwicklung}

Vergleicht man die Entwicklung intellektueller Fähigkeiten und basaler kognitiver Kompetenzen während der Schulzeit mit der Mannigfaltigkeit beruflicher Spezialisierungsnotwendigkeiten, so wird der Wechsel von der Allgemeinbildung zum Erwerb tätigkeitsspezifischer Expertise offenkundig. Durch viele wissenschaftliche Studien wird die alltägliche Erfahrung bestätigt, dass innerhalb der Grenzen breit definierter Begabungs- und Spezialbegabungsniveaus sehr unterschiedliche Wissensinhalte, Handlungsroutinen, methodische Kompetenzen und andere Verhaltensdispositionen erworben werden können.
Darf man unter diesen Umständen überhaupt eine Stabilität kognitiver Merkmale und langfristige Einflüsse juveniler Begabungsunterschiede auf die berufliche Entwicklung erwarten? Klassifiziert man Berufe nicht nach ihren spezifischen Tätigkeitsmerkmalen, sondern aufgrund ihrer geistigen Anforderungen, so erhält man auf die gestellte Frage eine interessante empirische Antwort, wie Abbildung 9 demonstriert (vgl. Weinert/Hany 2000).

Dargestellt ist ein Pfadmodell zur Vorhersage der intellektuellen und der beruflichen Entwicklung im Erwachsenenalter. Erwartungsgerecht ist die hohe Stabilität der Intelligenzunterschiede, wobei der etwas schwächere Zusammenhang zwischen den im 11. und im 40. Lebensjahr erhobenen Werten auf einen Wechsel des Messverfahrens in der GOLD-Studie zurückzuführen sein dürfte. Auffällig, aber erwartungsgemäß ist auch die Bedeutung des erreichten Bildungsniveaus für die berufliche Karriere, wobei die niedrigen numerischen Werte zwischen dem mit 25 und dem mit 40 Jahren erreichten Berufsstatus auf die Besonderheiten der Kriegsgeneration und ihrer beruflichen Entwicklung in der unmittelbaren Nachkriegszeit zurückzuführen sind.

Dass dabei die ökonomische Situation der Eltern sich differentiell auf die spätere ökonomische Situation der Kinder ausgewirkt hat, erstaunt nicht sehr. Interpretati-

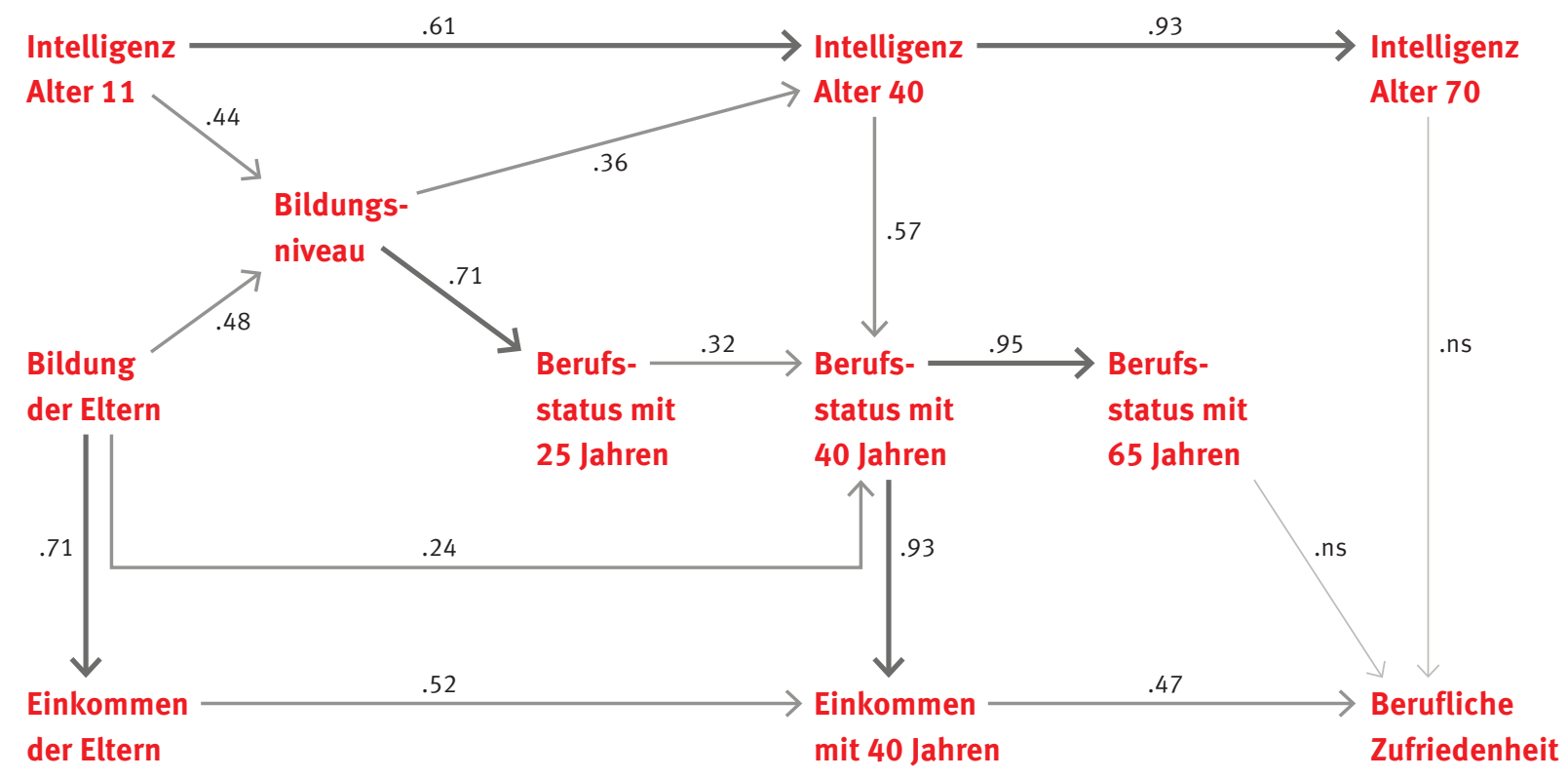

Abb. 9: Pfadmodell zur Vorhersage der intellektuellen und beruflichen Entwicklung. 
onsbedürftig ist eher die Tatsache, dass die berufliche Zufriedenheit im Rentenalter stärker von der ökonomischen Lage als vom früher erreichten Berufsniveau und von der intellektuellen Leistungsfähigkeit abhängig ist.

Dass die moderne Berufswelt weder dem Modell einer Kadettenanstalt noch einer philosophischen Gerechtigkeitstheorie entspricht, verdeutlichen die letzten zwei Schaubilder.

Während Abbildung 10 zeigt, dass Erwachsene mit hoher intellektueller Leistungsfähigkeit im Beruf durchweg erfolgreich sind, demonstriert Abbildung 11, dass unterdurchschnittlich intelligente Menschen eher schlechte berufliche Aussichten haben, es sei denn, ihre Eltern verfügten über einen hohen Bildungsgrad und über die damit im Durchschnitt verbundenen Privilegien. In diesem Fall sind die weniger intelligenten Kinder im späteren Erwachsenenalter fast ebenso erfolgreich wie die intelligenten Probanden. Ein Befund, der zweifellos im Einklang mit vielen Lebenserfahrungen steht, aber in ebenso vielen akademischen Oberseminaren zu heftigen Debatten führen dürfte.

Im Zusammenhang mit der GOLD-Studie haben mich in den letzten Jahren viele Journalisten gefragt, ob es nicht schrecklich sei, dass die Gene in so starkem Maße unser Lebensschicksal bestimmen. Ich konnte dem nie zustimmen! Ist es nicht letztlich eine List der Vernunft, dass etwa 50 Prozent der geistigen Unterschiede zwischen Menschen

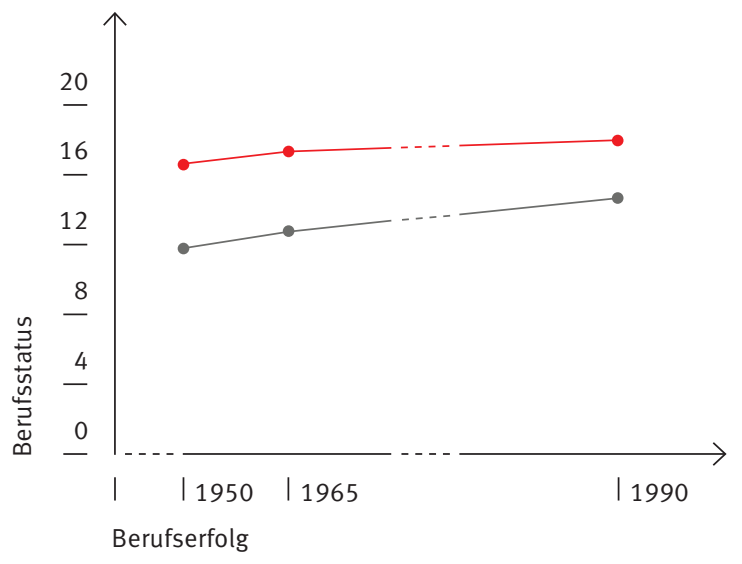

Abb. 10: Berufserfolg von überdurchschnittlich intelligenten Kindern, deren Eltern entweder über ein niedriges oder hohes Bildungsniveau verfügten.

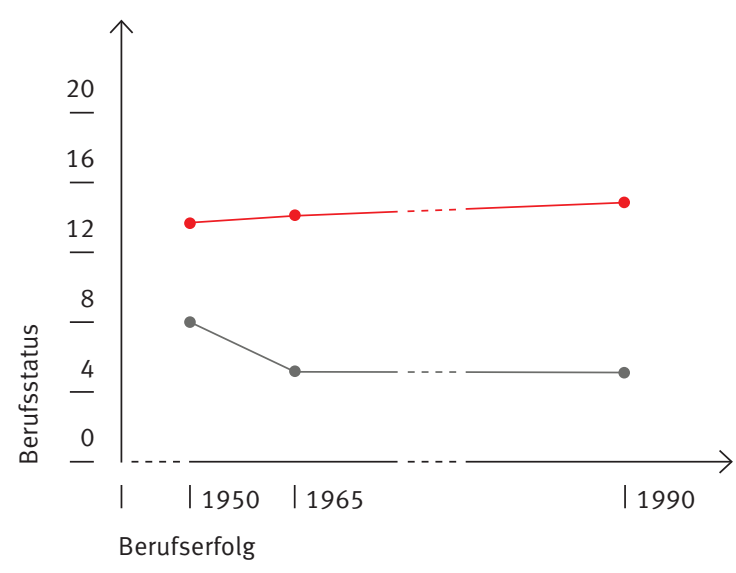

Abb. 11: Berufserfolg von unterdurchschnittlich intelligenten Kindern, deren Eltern entweder über ein niedriges oder hohes Bildungsniveau verfügten.

genetisch determiniert sind, ungefähr ein Viertel durch die kollektive Umwelt und ein weiteres Viertel durch die individuelle, zum Teil selbstgeschaffene Umwelt erklärbar sind? Man sollte dieses letzte Viertel weder kognitionspsychologisch noch motivationstheoretisch oder lebenspraktisch gering schätzen. Durch dieses komplexe Determinationsmuster geistiger Leistungsunterschiede zwischen verschiedenen Menschen erübrigen sich Schuldzuweisungen an die biologischen Eltern wie gegenüber der sozialen Umwelt. Es gibt aber auch keine Gründe für persönliche Resignation! Von der Anlage-Umwelt-Forschung aus betrachtet ist die Welt voller Spielräume für die geistige Entwicklung sehr unterschiedlich begabter Individuen. Ist das nicht beruhigend und motivierend zugleich? 


\section{Der Autor:}

Prof. Dr. Franz Emanuel Weinert (1930-2001) gehörte zu den einflussreichsten Fachvertretern der Psychologie im wissenschaftlichen und politischen Bereich. Seine Arbeits- und Forschungsschwerpunkte lagen in der kognitiven Entwicklung, insbesondere in der Entstehung individueller Intelligenz-, Motivations- und Persönlichkeitsunterschiede. Franz E. Weinert war 1980 bis 1986 Vizepräsident der Deutschen Forschungsgemeinschaft, 1984 bis 1986 Präsident der Deutschen Gesellschaft für Psychologie, 1990 bis 1999 Vizepräsident der Max-Planck-Gesellschaft und zudem Gründungsdirektor des Max-Planck-Instituts für psychologische Forschung in München. Von 1968 bis 1982 hatte er den Lehrstuhl für Entwicklungspsychologie und Pädagogische Psychologie an der Universität in Heidelberg inne. Zwischen 1990 und 1995 war Prof. Dr. Weinert Mitglied des Kuratoriums der Karg-Stiftung, 1999 und 2000 stellvertretender Stiftungsratsvorsitzender.

\section{Literatur}

Bloom, B.S. (1976): Human characteristics and school learning. New York: McGraw-Hill.

Dörfert, J. (1996): Dynamik und Genetik der Intelligenz. Göttingen: Cuvillierg.

Erdmann, K. D. (1969): Vorwort. In: Roth, H. (Hrsg.): Begabung und Lernen. Stuttgart: Klett, S. 5-6.

Herrnstein, R. J./Murray C. (1994): The Bell Curve. New York: The Free Press.

Plomin, R. (1988): The nature and nurture of cognitive abilities. In: Sternberg, R. J. (Hrsg.): Advances in the psychology of human intelligence. Bd. 4. Hillsdale: Erlbaum, S. 1-33.

Slavin, R.E. (1987): Mastery learning reconsidered. Review of Educational Research 57, S. 175-213.

Sternberg, R.J./Grigorenko, E. (Hrsg.) (1997): Intelligence, heredity, and environment. Cambridge: Cambridge University Press. Weinert, F.E./Geppert, U. (Hrsg.) (1998): Genetisch orientierte Lebensspannenstudie zur differentiellen Entwicklung (GOLD). Report Nr. 2: Erste Ergebnisse der Studie. München: Max-PlanckInstitut für psychologische Forschung.

Weinert, F.E./Hany, E.A. (2000): The role of intelligence as a major determinant of a successful occupational life. In: Van Lieshout, C.F.M./Heymans, P.G. (Hrsg.): Developing talent across the life-span. Hove: Psychology Press, S. 67-99.

Weinert, F.E./Helmke, A. (Hrsg.) (1997): Entwicklung im Grundschulalter. Weinheim: Beltz Psychologie Verlags Union.

Weinert, F. E./Schneider, W. (Hrsg.) (1999): Individual development from 3 to 12: Findings from the Munich Longitudinal Study. New York: Cambridge University Press. 